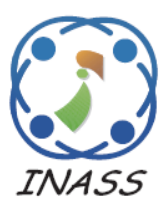

\title{
Guided Filtering Based Efficient Digital Differentiator Design for Electrocardiogram Signal Processing
}

\author{
Divya Jain $^{1 *} \quad$ Sanjaeev Narayan Sharma $^{1} \quad$ Alok Jain $^{1}$ \\ Department of Electronics and Instrumentation Engineering, \\ Samrat Ashok Technological Institute, Vidisha (M.P.), India \\ * Corresponding author’s Email divyajainphd@gmail.com
}

\begin{abstract}
Digital differentiation is an important phenomenon in digital signal processing to finding out sudden changes in signal. In this paper design of digital differentiator based on Fourier series expansion with quadratic fall in response has been presented. Error minimization criterion is applied to obtain minimum error in band of interest and to optimize the designed digital differentiator. Differentiator produces noise after differentiation and differentiated signal become noisy therefore guided filtering has been detailed to further smoothen the differentiated signal. Criterion for sampling frequency has been evaluated for particular cut-off frequency so that efficient selection of sampling frequency for Electrocardiogram (ECG) signal may possible. The results are demonstrated by applying designed differentiator on ECG signal and it has been found that the response of smoothened digital differentiator is equivalent to ideal differentiator. In this work sensitivity and error rate are 99.97 percent, and 0.069 percent, respectively.
\end{abstract}

Keywords: Digital differentiation, ECG, Signal smoothening.

\section{Introduction}

Digital signal processing (DSP) is an important mechanism which is used in various engineering problems. In many of these problems, time derivative of given signal is required [1,2]. This requirement leads to the development of digital differentiators. Digital differentiators (DD) have been widely used in signal processing, image processing, biomedical engineering, radar engineering, control systems, and other domains in recent years [3, 4]. This means differentiator is applicable in low frequency biomedical to high frequency radar and sonar application. Because of its wide varieties of applications, the design and implementation of digital differentiators has become a hot topic of research. [5-8]. Digital differentiators are used to compute the time-derivative of a realtime and/or stored signal, which needs high accuracy and a stable structural realization.

An ideal digital differentiator's frequency response is given by

$$
H_{d}\left(e^{j \omega}\right)=j \omega \pi \leq \omega \leq \pi
$$

where $j=\sqrt{-1}$ and $\omega \in[0, \pi]$ is the normalized frequency and $H_{d}$ is digital differentiator transfer function. An ideal differentiator has a constant phase response of $\pi / 2$, over the whole Nyquist frequency range. It is not possible to design an efficient DD in the entire band. Therefore, both low pass as well as wide band differentiators are designed. For the designing and implementation of digital differentiator various methods have been extensively examined in the literature. The design of a digital differentiator can be considered as four-step process. To begin, the digital differentiator's optimal frequency response is established. Secondly, choose between Finite Impulse Response (FIR) and Infinite Impulse Response (IIR) systems. Finally, the optimization method that will be utilised to compute the optimal system coefficients needs to be 


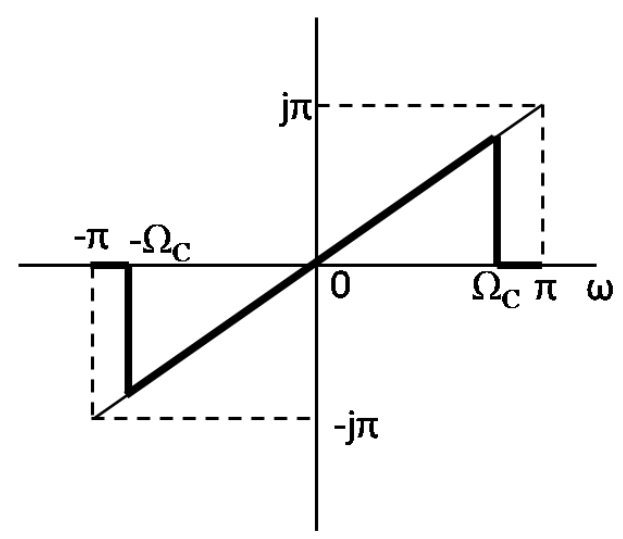

Figure. 1 Magnitude response of digital differentiator

considered. For the better understanding of digital filters, let us consider $x_{c}(t)$ a continuous time signal with its discrete version as $x_{d}(n T)$ where sampling period is $T, n$ is number of samples, and associated sampling frequency as $f_{s}$. If $f_{s}$ satisfies Nyquist criterion, than continuous time signal $\left(x_{c}(t)\right)$ can be constructed using samples values $x_{d}(n T)$ by using interpolation formula

$$
x_{c}(t)=\sum_{n=-\infty}^{n=\infty} x_{d}(n T) \frac{\sin \left[\frac{\pi}{T}\right](t-n T)}{\left[\frac{\pi}{T}\right](t-n T)}
$$

Therefore the derivative of $x_{c}(t)$ is not straight, and its derivative is corrupted with noise. Differentiator amplifies the high frequency noise this is the major problem related to differentiator design this problem increases with the increase of filter bandwidth and/or derivative order. Sampling frequency should be sufficiently high so that aliasing may not occur at the edges of the spectrum of sampled signal. Due to aliasing noise will be amplify after differentiation. Therefore, it is not possible to design efficient full band digital differentiator. Moreover, noise generated due to the quantization and external sources further degrades signal and its differentiation, so after differentiation smoothening of the signal is also required. The ideal frequency response as in Eq. (1), is not realizable using finite order filter. Moreover, at higher frequencies $(\omega \approx \pi)$ differentiation is only possible, when signal is noise and aliasing error free. On the other hand with IIR filter constant phase is not possible.

Many scholars have proposed several low pass and wideband recursive differentiators by efficiently approximating the ideal differentiator response in the whole Nyquist frequency range. The first order differentiation using central difference can be written as

$$
x^{\prime}[n]=\frac{x[n+1]-x[n-1]}{2}
$$

Taking the $\mathrm{z}$ transfrom we get,

$$
H[z]=\frac{z-z^{-1}}{2}
$$

Replacing $z=e^{j \omega n}$ tp obatin frequency domain transfer function as

$$
H\left[e^{j \omega n}\right]=\frac{e^{j \omega n}-e^{-j \omega n}}{2}=j \sin \omega n
$$

The generalized expression for different values of $n$ can be written as

$$
H[\omega]=j \sum_{n=1}^{N} C_{n} \sin \omega n
$$

In digital differentiation design, our objective is to minimize the difference between the transfer functions in Eqs. (4) and (1), by properly choosing order of the filter ' $n$ ' and coefficients $C_{n}$. It is also notable that the value of ' $n$ ' should be kept as small as possible. In the recent past, fractional order calculus (FOC) where fractional value of ' $n$ ' is used and coefficients are evaluated. The fractional order affects the coefficients of a non-causal finite impulse response (FIR) filter [9]. The fractionalorder differentiator (FOD) is considered in many applications like image processing $[10,11]$, control systems [12], signal processing [13, 14], and ECG signal processing applications based on the principle of fractional-order differentiation $[15,16]$. The main advantage of fraction order differentiation is its vast applicability on various problems as it can evaluate non-inter order derivatives. The main disadvantage is its incorrect differentiation for various functions. To overcome this limitation soft computing techniques are investigated for optimization of differentiator parameters. These soft computing algorithms are capable of delivering optimal coefficients in less time by minimising any multimodal error objective functions. The soft computing technique used are Simulated annealing (SA) [17], Genetic algorithm (GA) [18], Interior search algorithm [19] and particle swarm optimization [20] etc. However, it is also important to note that soft computing techniques are computationally complex and more costly.

The performance of the differentiator should also be observed on the chosen signal, to observe how differentiator performance on various points. In [21-23], ECG, R-peak detection using fractional order differentiation is presented. The other notable methods are also discussed in [24-26] for ECG peak and QRS complex detections.

In this paper, a low pass differentiator design is 
proposed. The cut-off frequency of the differentiator is selected in such a way that error can be kept within acceptable range and filter fall is sharp at corner frequencies. In many medical applications noise of differentiation is not acceptable; therefore various classes of filter design and optimization process are proposed. For the smoothening of the ECG signal guided filtering is considered. The main advantages of the proposed mechanism are

1. Differentiation is noise free.

2. It is a finite order filter, thus can be easily realizable.

3. Filter can easily be designd for various applications by properly choosing signal sampling frequency $\left(f_{s}\right)$.

The rest of the paper, is organised as follows, in section 2, basics of digital differentiation is discussed. Proposed differentiator design is presented in section 3 of the paper. The results are proposed in section 4, and finally, in section 5 major conclusions of the paper are discussed.

\section{Basic of digital differentiation}

The frequency response of ideal digital differentiator is given by

$$
\begin{array}{ll}
H_{d}\left(e^{j \omega}\right)=j \omega & 0 \leq \omega \leq \pi \\
H_{d}\left(e^{j \omega}\right)=-j \omega & \pi-\leq \omega \leq 0
\end{array}
$$

Hence, the ideal impulse response can be obtained by taking inverse DTFT and is given by

$$
h_{d}(n)=\frac{1}{2 \pi} \int_{-\pi}^{\pi} H_{d} e^{j \omega n} d \omega
$$

Inserting Eq. (7) in Eq. (8) and after integration we get,

$$
h_{d}(n)=\frac{\cos \pi n}{n}
$$

$h_{d}(n)$ has two sided impulse response of infinite length, which is practically unrealizable. It should be noted that $h_{d}(n)=-h_{d}(-n)$.Thus differentiator has asymmetric impulse response about point $n=0$, to make it realizable we shift impulse response towards right by an amount $\gamma=$ $\frac{(N-1)}{2}$. The impulse response of the differentiator about $n=\alpha$ is

$$
h_{d}(n)=\frac{\cos \pi(n-\gamma)}{(n-\gamma)}-\infty<n<\infty, n \neq \gamma
$$

Windowing functions are used, to control the response of the filters. In digital filters windowing method is used to convert infinite impulse response to finite impulse response. In case of windowing the impulse response is written as

$$
h(n)=h_{d}(n) w(n) \quad 0<n<N-1
$$

Inserting Eq. (10) in Eq. (11) we get,

$$
h(n)=\frac{\cos \pi(n-\gamma)}{(n-\gamma)} \times w(n) 0<n<N-1, n \neq \gamma
$$

As $h(n)$ is asymmetric about $n=\gamma$, thus $n$ has to be an odd integer only. The magnitude response for odd $N$ follows $h(n)=-h(N-1-n)$. This is type III linear phase FIR differentiator. This filter is not a full band filter as at $\pi$ response is zero.

\section{Proposed Method}

In this section the proposed differentiator design is presented, which can be efficiently used in finite band with cut-off frequency $\Omega_{c}$. Considering Fig. 1, the ideal differentiator can be approximated as triangular function

$$
H_{d}\left(e^{j \omega}\right)=j \omega-\Omega \leq \omega \leq \Omega
$$

where, $\Omega$ is angular sampling frequency, and relate with sampling interval $T$ as $T=2 \pi / \Omega$. The Fourier series expansion can be written as

$$
H_{d}\left(e^{j \omega}\right)=H_{d}(j \omega+j \Omega)=\sum_{n=-\infty}^{n=\infty} h_{n} e^{-j n \omega T}
$$

and the impulse response can be obtained as

$$
h_{d}(n)=\frac{1}{\Omega} \int_{-\Omega / 2}^{\Omega / 2} H_{d} e^{j \omega n T} d \omega
$$

Inserting Eq. (13), in above and solving the integral we get,

$$
h_{d}(n)=\frac{\cos \left(\frac{n \Omega T}{2}\right)}{n T}-\frac{2 \sin \left(\frac{n \Omega T}{2}\right)}{\Omega(n T)^{2}} \quad \text { if } n \neq 0
$$

But as discussed, differentiation in the entire band is not possible let the cut-off frequency beyond which differentiation is not possible is $\Omega_{\mathrm{C}}$. The impulse response within the cut-off frequencies can be obtained as

$$
h_{d}(n)=\frac{\cos \left(\frac{n \Omega_{\mathrm{c}} T}{2}\right)}{n T}-\frac{2 \sin \left(\frac{n \Omega_{\mathrm{c}} T}{2}\right)}{\Omega_{\mathrm{c}}(n T)^{2}} \quad \text { if } n \neq 0
$$

Using the shifting property (Eq. (10)) we get 


$$
h_{d}(n)=\frac{\cos \left(\frac{(n-\gamma) \Omega_{\mathrm{c}} \pi}{\Omega}\right)}{2(n-\gamma) \pi / \Omega}-\frac{2 \sin \left(\frac{(n-\gamma) \Omega_{\mathrm{c}} \pi}{\Omega}\right)}{\Omega_{\mathrm{c}}(2(n-\gamma) \pi / \Omega)^{2}} \text { if } n \neq \gamma
$$

In the entire band $\Omega=\Omega_{C}$ and $\Omega=2 \pi$, the Eq. (14) is same as Eq. (6). However to take advantage of sharp decay of second term we should consider $\Omega<2 \pi$, which can be realized windowing function and cutoff frequency can be chosen using energy minimization criterion as explained in the next section.

\section{Optimization of proposed differentiator}

For smaller values of frequencies the filter response is very close to ideal response. However as frequency increases the difference between ideal and actual response increases, and mean square error also increases. The mean square error is defined as:

$$
E(\omega)=\int_{-\pi}^{\pi}\left|H_{d}(\omega)-H(\omega)\right|^{2} d \omega
$$

Error is huge in full band, therefore considering error within the cut-off frequency, therefore

$$
E(\omega)=\int_{-\Omega_{c}}^{\Omega_{c}}\left|H_{d}(\omega)-H(\omega)\right|^{2} d \omega
$$

Substituting expressions of transfer functions

$$
E(\omega)=\int_{-\Omega_{c}}^{\Omega_{c}}\left|j \omega-j \sum_{n=1}^{N} C_{n} \sin \omega n\right|^{2} d \omega
$$

After solving integrals we get,

$$
\begin{array}{r}
E(\omega)=\frac{2}{3} \Omega_{c}^{3}+\Omega_{\mathrm{c}} \sum_{\mathrm{n}=1}^{\mathrm{N}} C_{\mathrm{n}}^{2}-\frac{1}{2} \sum_{\mathrm{n}=1}^{\mathrm{N}} C_{\mathrm{n}}^{2} \frac{\sin 2 \Omega_{\mathrm{c}} \mathrm{n}}{\mathrm{n}}+ \\
4 \sum_{\mathrm{n}=1}^{\mathrm{N}} \frac{\mathrm{C}_{\mathrm{n}}^{2}}{\mathrm{n}^{2}}\left[\Omega_{\mathrm{c}} \mathrm{n} \cos \Omega_{\mathrm{c}} \mathrm{n}-\sin \Omega_{\mathrm{c}} \mathrm{n}\right]
\end{array}
$$

Further, from Eq. (6), we have

$$
\frac{d H[\omega]}{d \omega}=\frac{d}{d \omega} j \sum_{n=1}^{N} C_{n} \sin \omega n=j \sum_{n=1}^{N} n C_{n} \cos \omega n
$$

And from Eq. (1) we have,

$$
\frac{d H_{d}(j \omega)}{d \omega}=j
$$

Equating both the Eqs. (23) and (24), we have

$$
\left|\frac{d H_{d}(j \omega)}{d \omega}\right|_{\omega=0}=\left|\frac{d H(j \omega)}{d \omega}\right|_{\omega=0}=\sum_{n=1}^{N} n C_{n}=1
$$

Now choosing, $\Omega_{c}=\alpha \pi \quad 0<\alpha<1$

$$
\begin{aligned}
& E(\omega)=\frac{2}{3}(\alpha \pi)^{3}+\alpha \pi \sum_{\mathrm{n}=1}^{\mathrm{N}} \mathrm{C}_{\mathrm{n}}^{2}- \\
& \frac{1}{2} \sum_{\mathrm{n}=1}^{\mathrm{N}} \mathrm{C}_{\mathrm{n}}^{2} \frac{\sin 2 \alpha \pi \mathrm{n}}{\mathrm{n}}+ \\
& \quad 4 \sum_{\mathrm{n}=1}^{\mathrm{N}} \frac{\mathrm{C}_{\mathrm{n}}^{2}}{\mathrm{n}^{2}}[\alpha \pi \mathrm{n} \cos \alpha \pi \mathrm{n}-\sin \alpha \pi \mathrm{n}]
\end{aligned}
$$

Referring Eq. (25) and $n=1$ we get $C_{1}=1$. The Eq. (26), simplifies to

$$
\begin{aligned}
& E(\omega)=\frac{2}{3}(\alpha \pi)^{3}+\alpha \pi-\frac{1}{2} \sin 2 \alpha \pi+[\alpha \pi \cos \alpha \pi- \\
& \sin \alpha \pi]
\end{aligned}
$$

But it should be noted that, with rise in $\alpha$ error increases. Therefore, error is kept to a desired value and then $\alpha$ is finalized. In Fig. 2, absolute error vs. $\alpha$ is plotted for various values of $\alpha$, the absolute error for $\alpha$ equals 0.16 and error is 0.0102 . It is should be noted that this is the error this covers whole frequency range where absolute error is minimum. However, as we are interested in low frequency regime, therefore we can select $\alpha$ such that in low frequency regime error is lesser at the expense of higher error in higher frequency regime.

Considering Eq. (6), multiplied by 2 and with constraints Eq. (24) and (25), we obtain following sets of equations.

$j \sum_{n=1}^{2} C_{n} \sin (0)=0 \quad$ or $\quad 0=0$ $2 j \cos (0) \sum_{n=1}^{2} n C_{n}=j$ or $2 C_{1}+4 C_{2}=1$ $2 j \sum_{n=1}^{2} C_{n} \sin (n \pi)=0$ or $\quad 0=0$ $2 j \sum_{n=1}^{2} n C_{n} \cos n \pi=j$ or $-2 C_{1}+4 C_{2}=1$

Solving we get, $c_{1}=1 / 2$ and $c_{2}=1 / 4$. Similarly for higher values of $N$ coefficients can be evaluated. In Fig. 3, transfer functions for ideal and approximated

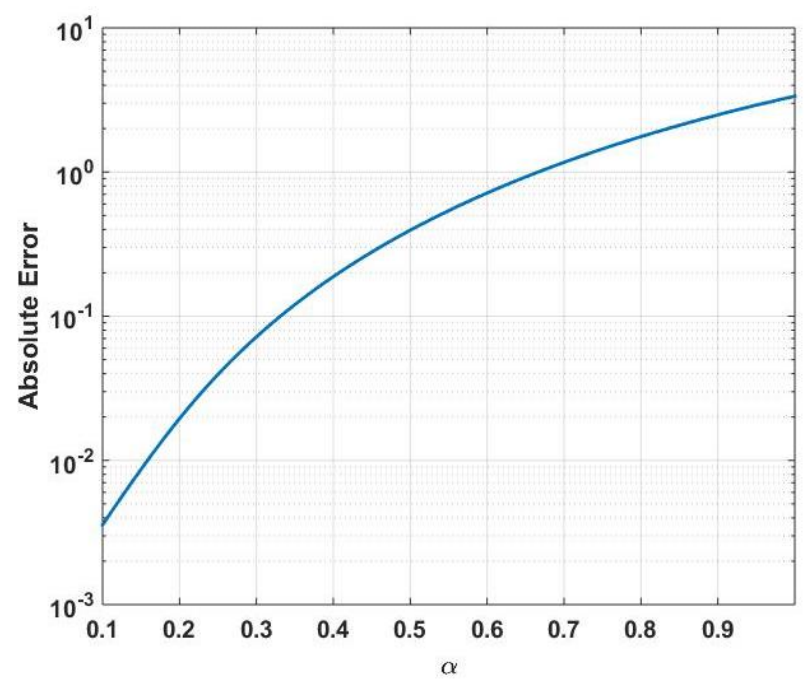

Figure. 2 Absolute error vs. $\alpha$ 


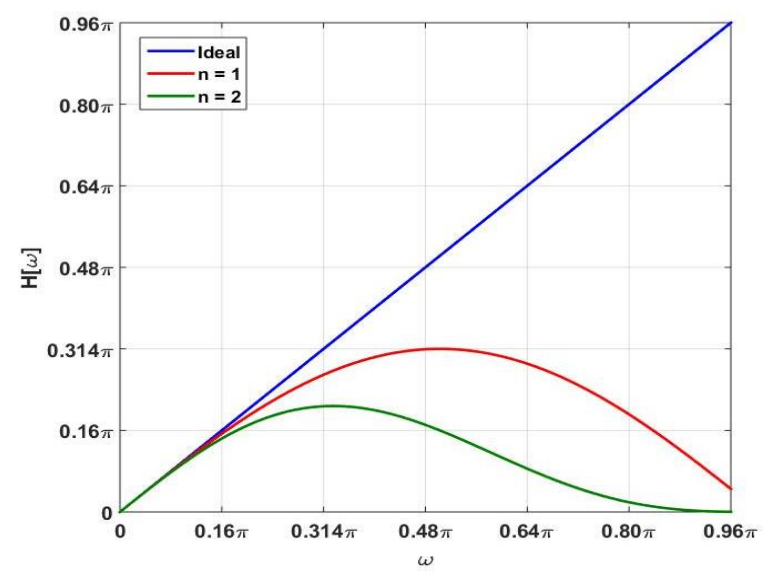

Figure. 3 Differentiator characteristic for $n=1,2$ and ideal

differentiators are shown. In the low frequency regime $\omega \approx 0.16$, for $n=1,2$ and ideal approximated differentiators are very much similar to ideal one. For proper differentiation it is required that it should be symmetric about peak value (in above about $\omega \approx 0.16$ ), otherwise differentiators will be noisy. It is clear from the above figure that as ' $n$ ' increases cutoff frequency decreases and approximated differentiators gets closer to ideal differentiator.

In this paper digital differentiation is designed for ECG signal differentiation. Let us assume that ECG signal is a low pas signal with cut-off frequency $f_{c}$, then to avoid aliasing sampling frequency $\left(f_{s}\right)$ must be twice of $f_{c}$. The digital differentiator frequency would be

$$
\omega_{c}=2 \pi \frac{f_{c}}{f_{s}}
$$

If $\Omega_{c}=\alpha \pi \quad$ then we can write

$$
\alpha \pi=2 \pi \frac{f_{c}}{f_{s}} \quad \text { or } f_{s}=2 \frac{f_{c}}{\alpha}
$$

Therefore, for ECG signal sampling frequency can be selected efficiently. due to the respiration and muscles contraption/expansion, minor variations are observed in recorded ECG and when ECG signal is differentiated these minor variations appear as noise. To remove such noise smoothed filters can be used. There are various mechanisms which can smoothen the ECG noise. In this work we have considered the guided filter.

\section{Guided image smoothening}

For an image $\left(I_{n}\right)$ the output $\left(I_{n}^{\prime}\right)$ of guided filtering is a linear transformation of guided image ' $G_{n}$ ' which is written as [27].

$$
I_{n}^{\prime}=a_{i} G_{n}+b_{i} \quad \forall n \in w_{i}
$$

$a_{i}$ and $b_{i}$ are the co-efficient in window $w_{i}$. The guided image filtering problem can be expressed as the reduction of the difference between the input and output data, where $\varepsilon$ is the smoothness parameter that determines the degree of smoothness. The minimization problem is formulated as

$$
E\left(a_{i}, b_{i}\right)=\sum_{n \in w_{i}}\left[\left(a_{i} G_{n}+b_{i}-\mathrm{I}_{\mathrm{n}}\right)^{2}+\varepsilon a_{i}^{2}\right]
$$

After carrying out multiple calculations, the coefficient are evaluated as

$a_{i}=\frac{\frac{1}{|w|} \sum_{n \in w_{i}} G_{n} I_{n}-G_{i}^{m} I_{i}^{m}}{\sigma_{i}^{2}+\varepsilon}$ and $b_{i}=I_{i}^{m}-a_{i} G_{i}^{m}$

where, mean values of the related parameter are represented by the bar. $|w|$ denotes the total number of pixels in window $w_{i}$

$$
I_{n}^{\prime}=\left(\frac{1}{|w|} \sum_{i \in w_{n}} a_{i}\right) G_{n}+\left(\frac{1}{|w|} \sum_{i \in w_{n}} b_{i}\right)
$$

The guided filtering weight function $\left(W_{n m}^{G F}(G)\right)$ can also be expressed as [27]

$$
W_{n m}^{G F}(G)=\frac{1}{|w|^{2}} \sum_{i:(n, m) \in w_{i}}\left(1+\frac{\left(G_{n}-G_{i}^{m}\right)\left(G_{m}-G_{i}^{m}\right)}{\sigma_{i}^{2}+\varepsilon}\right)
$$

The noise smoothened ECG is given by

$$
I_{n}^{\prime}=\sum_{m \in w_{n}} W_{n m}^{G F}(G) I_{m}
$$

\section{Results}

The Table 1. shows comparative results in terms of error for ideal and proposed differentiators. Alaoui et. al. [29], used GA and SA for the coefficient optimization and calculate absolute errors of 2.0848 and 1.5966 respectively. Gupta et.al, considered PSO with error of 36.687 which is huge. Further, Kumar et.al considered ISA and limited to error to a very low value of 1.6091. In this work, with the proposed method error is reduced to 0.0102 , which is better among the comparative recent methods.

Table 1. Comparison of error with recent methods

\begin{tabular}{|l|l|l|}
\hline Reference & Method & Error \\
\hline Alaoui et.al.[30] & GA & 2.0848 \\
\hline Alaoui et.al. [30] & SA & 1.5966 \\
\hline Gupta et al. [31] & PSO & 36.687 \\
\hline Kumar et.al [19] & ISA & 1.6091 \\
\hline Jain et.al. & Proposed & 0.0102 \\
\hline
\end{tabular}


The differentiation using proposed method is evaluated using the MIT-BIH arrhythmia database [28], where the ECG record is 30 minutes long and sampled at $360 \mathrm{~Hz}$. The sample ECG data is downloaded from https://physionet.org/physiobank/database/. In the experiment a 1000 points ECG is taken with peak value of 187 and minimum value of 16 (Fig. 4). The sample points are shown on ' $\mathrm{X}$ ' axis, and on ' $\mathrm{Y}$ ' axis amplitude in $(\mathrm{mV})$ is shown. The ECG is signal is corrupted with additive noise with maximum amplitude variation of \pm 10 . The noisy ECG is also shown, it is clear that noise is more dominant around baseline.

As we saw previously, differentiation introduces noise; therefore in the next step smoothening is applied on noisy differentiated ECG signal. The noise in ECG is added due to quantization, noise of channel, noises due to the muscle movements etc [29]. The input and noisy ECGs are same as in Fig. 4. It is further clear from the Fig. 5 that differentiation leads to the accumulation of noise. It is also noticeable that smoothening reduces noise significantly.

In Fig. 6, ideal and digital differentiation is shown along with differentiated smoothened signal (proposed work). It is very much clear from the figure that, due to the limitations of digital differentiators after differentiation smoothening of signal is necessary. Fig. 7, is the zoomed version of Fig. 6, and it clearly, shows that digital differentiation with smoothening function is equivalent to the ideal differentiator. The main advantage of guided filtering is that it also smoothen the signals at the corner or kink where most of the traditional smoothening method fails.
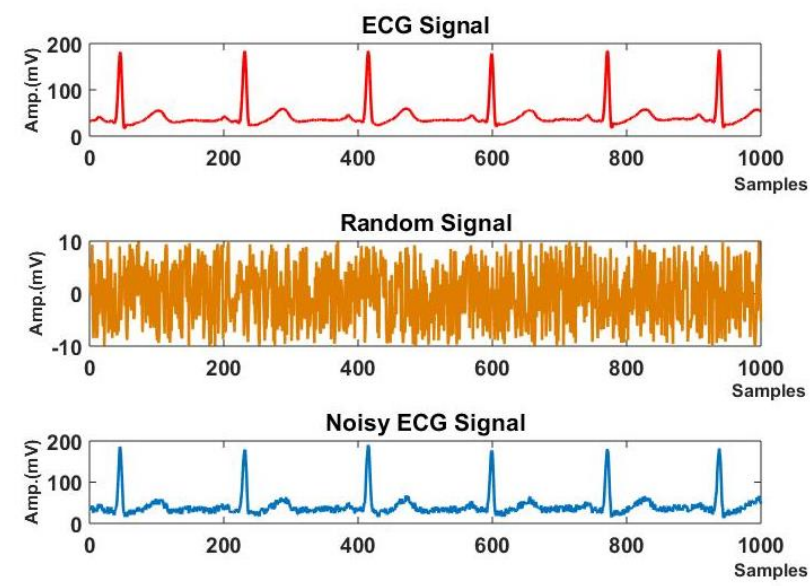

Figure. 4 ECG input, noise and noise added signal
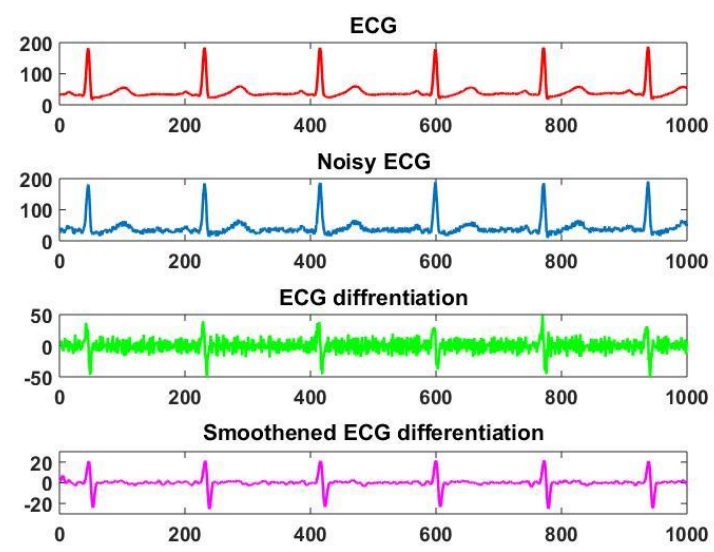

Figure. 5 Noisy ECG differentiation and smoothening

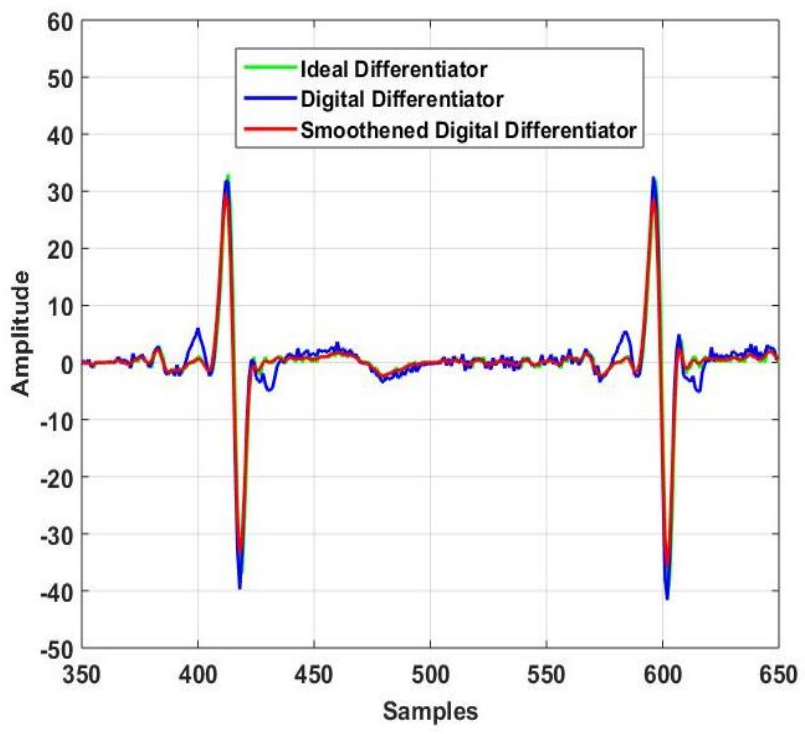

Figure. 6 Digital differentiator comparisons

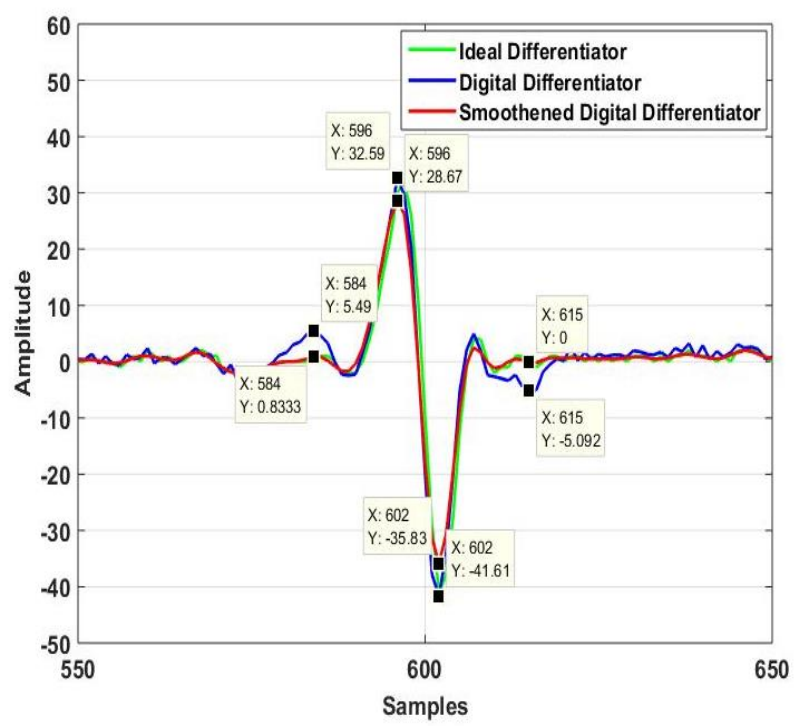

Figure. 7 Digital differentiator comparisons 


\begin{tabular}{|l|l|}
\hline $\begin{array}{l}\text { True } \\
\text { Positives }\end{array}$ & $\begin{array}{l}\text { False } \\
\text { Positives }\end{array}$ \\
\hline $\begin{array}{l}\text { True } \\
\text { Negatives }\end{array}$ & $\begin{array}{l}\text { False } \\
\text { Negatives }\end{array}$ \\
\hline
\end{tabular}

Figure. 8 Confusion matrix

Table 2. Comparison of notable methods (Sensitivity and Error rate)

\begin{tabular}{|c|l|c|}
\hline Methods & $\begin{array}{c}\text { Sensitivity } \\
\text { S (\%) }\end{array}$ & $\begin{array}{c}\text { Error rate } \\
\text { ER (\%) }\end{array}$ \\
\hline Ferdi et.al [21] & - & 0.37 \\
\hline Benmalek et.al [22] & 99.86 & 0.28 \\
\hline Kaur et.al [23] & 99.93 & 0.117 \\
\hline Sabberwal et.al [24] & 99.9 & 0.16 \\
\hline Sabberwal et.al [25] & 99.9 & 0.135 \\
\hline Kaur et.al [26] & 99.95 & 0.095 \\
\hline Proposed algorithm & 99.97 & 0.069 \\
\hline
\end{tabular}

The performance evaluation of the differentiator on signal is done based on peak detection. The correct detection of actual peaks is denoted by (TP), non-detection of actual peaks is denoted by (TN), detection of incorrect peaks is denoted by (FP) and non detection of incorrect peaks is denoted by (FN).

The Sensitivity is defined as

$$
S=\frac{T P}{T P+F N} \text { and } E R=\frac{F P+F N}{T P+T N+F P+F N}
$$

In Table 2, sensitivity and error rates are shown for recent methods. It can be observed that sensitivity is more than $99 \%$ for all the compared methods, in case of Kaur et.al [26] the sensitivity is $99.95 \%$. However, error rate varies significantly. For Ferdi et.al and Benmalek et.al works error is comparatively larger. In case of fractional order $(p)$ filter for lower value of ' $p$ ' artefacts peaks are present, while for higher order of ' $p$ ' some information is lost [26]. In our proposed method artefacts peaks are eliminated using guided image filtering, which is not possible in average smoothening as in [26].

Therefore it can be concluded that the proposed digital filter design is superior to recently proposed digital filter design.

\section{Conclusions}

In this paper description about digital filtering process is presented. This paper lay down the fundamental processes in digital differentiation. A method is proposed using the Fourier series expansion for digital differentiation, and advantage of proposed method is detailed. Simplified method based on anti-symmetry of III order differentiation which is based on least square error is also detailed and important concepts are discussed. Finally smoothing of digital differentiation is discussed using guided filtering which is found to be a necessary operation in case of digital differentiation. In this work sensitivity and error rate are 99.97 percent, and 0.069 percent, respectively. The current technique outperformed other commonly used approaches in the literature. In future work, we aim to increase the cut-off frequency of the differentiator, still maintaining lower error.

\section{Conflicts of interest}

The authors declare no conflict of interest.

\section{Author contributions}

Abstract, Introduction, Basics of differentiation, Proposed work, Results, and Conclusions were equally contributed by Divya Jain, Sanjaeev Narayan Sharma and Alok Jain.

\section{Acknowledgments}

This work was not supported by any organization.

\section{References}

[1] J. Proakis, "Digital signal processing: principles algorithms and applications" Pearson Education India, 2001.

[2] M. A. A. Alaoui, "Novel approach to designing digital differentiators", Electronics Letters, Vol. 28, No. 15, pp. 1376-1378, 1992.

[3] J. Luo, K. Ying, P. He, and J. Bai, "Properties of Savitzky-Golay digital differentiators", Digital Signal Processing, Vol. 15, No. 2, pp. 122-136, 2005.

[4] S. Ari, M. K. Das, and A. Chacko, "ECG signal enhancement using S-Transform", Computers in Biology and Medicine, Vol. 43, No. 6, pp. 649-660, 2013.

[5] M. A. A. Alaoui, "Class of digital integrators and differentiators", IET Signal Processing, Vol. 5, No. 2, pp. 251-260, 2011. 
[6] M. A. A. Alaoui, "Linear phase low-pass IIR digital differentiators", IEEE Transactions on Signal Processing, Vol. 5, No. 2, pp. 697-706, 2007.

[7] M. Jain, M. Gupta, and N. Jain, "Linear Phase Second Order Recursive Digital Integrators and Differentiators", Radioengineering, Vol. 21, No. 2, pp. 712-717, 2012.

[8] C. C. Tseng, "Design of fractional order digital FIR differentiators", IEEE Signal Processing Letters, Vol. 8, No. 3, pp. 77-79,2001.

[9] Y. Ferdi, "Improved lowpass differentiator for physiological signal processing", In: Proc. of International Symposium on Communication Systems, Networks \& Digital Signal Processing (CSNDSP 2010), pp. 747-750, 2010.

[10] S. Kumar, R. Saxena, and K. Singh, "Fractional Fourier transform and fractional-order calculusbased image edge detection", Circuits, Systems, and Signal Processing, Vol. 36, No. 4, pp. 1493-1513, 2017.

[11] Y. Pu, J. Zhou, and X. Yuan, "Fractional differential mask: a fractional differential-based approach for multiscale texture enhancement", IEEE Transactions on Image Processing, Vol. 19, No. 2, pp. 491-511, 2009.

[12] Y. Luo, Y. Chen, H. S. Ahn, and Y. G. Pi, "Fractional order periodic adaptive learning compensation for state-dependent periodic disturbance", IEEE Transactions on Control Systems Technology, Vol. 20, No. 2, pp. 465472, 2011.

[13] S. Kumar, K. Singh, and R. Saxena, "Analysis and design of non recursive digital differentiators in fractional domain for signal processing applications", PhD diss. 2014.

[14] K. Singh, R. Saxena, and S. Kumar, "Caputobased fractional derivative in fractional Fourier transform domain", IEEE Journal on Emerging and Selected Topics in Circuits and Systems, Vol. 3, No. 3, pp. 330-337, 2013.

[15] G. B. Moody and R. G. Mark, "The impact of the MIT-BIH arrhythmia database", IEEE Engineering in Medicine and Biology Magazine, Vol. 20, No. 3, pp. 45-50, 2001.

[16] M. D. Ortigueira, "Fractional calculus for scientists and engineers", Springer Science \& Business Media, Vol. 84, 2011.

[17] R. Lahcene, A. Sebbane, and K. Aissa, "Optimal tuning of fractional order PID controller for AVR system using simulated annealing optimization algorithm", In: Proc. of International Conference on Electrical Engineering-Boumerdes (ICEE-B), pp. 1-6. 2017.
[18] S. T. Tzeng and H. C. Lu, "Genetic algorithm approach for designing higher-order digital differentiators", Signal Processing, Vol. 79, No. 2, pp. 175-186, 1999.

[19] M. Kumar, T. K. Rawat, A. Jain, A. Singh, and A. Mittal, "Design of digital differentiators using interior search algorithm", Procedia Computer Science, Vol. 57, pp. 368-376, 2015.

[20] M. K. Jallouland and M. A. A. Alaoui, "Design of recursive digital integrators and differentiators using particle swarm optimization", International Journal of Circuit Theory and Applications, Vol. 44, No. 5, pp. 948-967, 2016.

[21] Y. Ferdi, J. P. Herbeuval, A. Charef, and B. Boucheham, " $\mathrm{R}$ wave detection using fractional digital differentiation", ITBM-RBM, Vol. 24, No. 5-6, pp. 273-280, 2013.

[22] M. Benmalek, A. Charef, "Digital fractionalorder operators for R-wave detection in electrocardiogram signal", IET Signal Processing, Vol. 3, No. 5, pp. 381-390, 2009.

[23] A. Kaur, S. Kumar, A. Agarwal, and R. Agarwal, "An efficient R-peak detection using Riesz fractional-order digital differentiator", Circuits, Systems, and Signal Processing, Vol. 39, No. 4 pp. 1965-1987, 2020.

[24] P. Sabherwal, M. Agrawal, and L. Singh, "Automatic detection of the $\mathrm{R}$ peaks in singlelead ECG Signal”, Circuits System and Signal Processing, Vol. 36, No. 11, pp. 4637-4652, 2017.

[25] P. Sabherwal, L. Singh, and M. Agrawal, "Aiding the detection of QRS complex in ECG signals by detecting S peaks independently", Cardiovasc. Eng. Technol., Vol. 9, pp. 1-13, 2018.

[26] A. Kaur, R. Agarwal, S. Agarwal, and A. Kumar, "A Novel approach to ECG R-peak detection", Arabian Journal of Science and Engineering, Vol. 44, No. 8, pp. 6679-6691, 2019.

[27] He, Kaiming, J. Sun, and X. Tang, "Guided image filtering", IEEE Transactions on Pattern Analysis and Machine Intelligence, Vol. 35, No. 6, pp. 1397-1409, 2012.

[28] B. G. Moody and R. G. Mark, "The impact of the MIT-BIH arrhythmia database", IEEE Engineering in Medicine and Biology Magazine, Vol. 20, No. 3, pp. 45-50, 2001.

[29] D. Jain, S. N. Sharma, and A. Jain, "Electrocardiogram transmission over OFDM system", Journal of Optical Communications, ahead of print, 2021. 
[30] A. Alaoui, M. A, and M. Baydoun, "Novel wide band digital differentiators and integrators using diơerent optimization techniques", In: Proc. of International Symposium on Signals, Circuits and Systems, pp. 1-4, 2013.

[31] M. Gupta, B. Relan, R. Yadav and V. Aggarwal, "Wideband digital integrators and differentiators designed using particle swarm optimisation", IET Signal Processing, Vol. 8, No. 6, pp. 668-679, 2014. 